\title{
Locating Facilities Under Deliberate Disruptive Attacks
}

\author{
Anton V. Ushakov ${ }^{(\varpi)}$ (D) and Igor Vasilyev(0) \\ Matrosov Institute for System Dynamics and Control Theory of SB RAS, \\ 134 Lermontov str., 664033 Irkutsk, Russia \\ \{aushakov, vil\}@icc.ru \\ http://iv.icc.ru
}

\begin{abstract}
Facility disruptions or failures may occur due to natural disasters or a deliberate man-made attack. Such an attack is known as interdiction. Recently, facility location problems, addressing intentional strikes against operating facilities and strategies to reduce their impact, have received particular attention. In this paper, we present a new location-interdiction median problem aimed at designing a distribution network which is robust to the worst-case, long-term facility losses. We suppose that there are two players: defender (system designer) and attacker. The defender decides where to locate facilities to minimize the overall cost of supplying the demands of customers. The attacker determines which $r$ facilities to interdict to maximize the cost of serving the customers from the remaining operational facilities. Note that we suppose that the facilities are attacked simultaneously and interdicted facilities become unavailable. We propose bilevel and single-level integer formulations of this problem. For a particular case when the attacker hits a single facility, we develop a fast local search procedure based on implicit enumeration of interdiction strategies. We test our approaches in a series of computational experiments on well-known test problems.
\end{abstract}

Keywords: Interdiction $\cdot$ Bilevel programming $\cdot$ p-median problem $\cdot$ Facility location $\cdot$ Disruption $\cdot$ Integer programming

\section{Introduction}

Facility location problems are ones of the most widely studied problems in combinatorial optimization and integer programming. Facilities and the corresponding connecting infrastructure are the main ingredients in any production, distribution, or service system and supply chains [29]. A traditional approach to the design of such systems assumes that all their components always remain operable. However, some accidents, like natural disasters or man-made attacks, can substantially reduce the system efficiency and even make it incapacitated. For example, the current coronavirus outbreak is disrupting the global supply chains from tech industry (production of cell phones and electronics) to fashion and car 
manufacturing. Hyundai and Nissan announced that they would stop manufacturing in South Korea, Europe, and the US because of a lack of parts from China [5,8]. Meanwhile, Tesla considers to delay the production in Shanghai for at least one week. The disruptions can also be man-made. For example, the current French pension reform strike is the longest French strike over the last 50 years, which results in billion losses.

Given a service system, one may expect two main types of disruptions: facility disruptions (loss of production capabilities) and failures of links connecting facilities and customers. In the field of facility location, reliability and sustainability issues of service systems are extensively studied in the scope of the so-called reliable facility location. Such kind of models usually assume that the system components have some inherent probability of failure. Thus, the aim is to maximize the expected system efficiency, e.g. minimize the expected service cost in case of possible failures. The stochastic approach may be quite useful when modeling sudden losses of components due to natural disasters or some inherent system failures. However, disruptions may be caused not only by natural disasters (like earthquakes or fire) but also by disruptive man-made actions (sabotage, deliberate attacks). Intentional attack on a supply network is also called interdiction [11]. Interdiction models are aimed at identifying critical infrastructure elements such that the loss of them due to an attack causes the maximum possible harm and makes a system much less efficient. Originally, such models were mostly studied in military planning applications. Indeed, it is natural to identify the places for interdicting enemies supply lines in order to inflict the maximal damage. The first interdiction problem was considered in [22] where the aim of interdiction was to disrupt enemy supply flows with a limited budget available [35]. Note that most of the military-induced applications are focused on interdicting links of transportation networks (e.g. shortest paths) [18].

Recently, the interest has revived for interdiction problems due to fast growing threat of terrorist attacks (especially, after the September 11 attacks). Nowadays, we are witnessing how vulnerable may be facilities to such threats. International counter-terrorism efforts are forcing the terrorist groups, like al Qaeda, transfer their attention from so-called hard targets (embassies and military assets) to soft targets (civilian facilities). The main goal of such attacks is to engender the largest possible harm. For a service system, the harm is viewed as the loss of service coverage or efficiency (increase in the overall service costs). The first facility interdiction models were addressed in [11] where the $r$-interdiction median and covering problems were proposed. For example, the r-interdiction median problem is to find which $r$ existing facilities to remove in order to decrease the efficiency of the existing supply system the most.

The next research in this field was focused on developing strategies of hedging against the worst-case attacks. Thus, in [10], the authors extend the $r$ interdiction problem by the possibility of fortifying (protecting) some existing facilities so that they become immune to attacks. The problem is then modeled as a bilevel integer program involving two players: attacker and defender [27]. This problem has received great attention and a number of effective solution 
algorithms has been developed. Among most effective are the implicit enumeration algorithm [27], an exact approach based on reformulating the problem as the maximal covering problem [28,36], and a branch-and-cut algorithm from [26].

Numerous papers addressing various bilevel facility location protection-interdiction problems have been published since then, e.g. hub interdiction median problems $[15,24]$, the stochastic r-interdiction median problem with fortification [19], a $r$-interdiction median problem involving a defender's budget for fortification and penalties for serving the demands of customers from interdicted facilities [4], a partial interdiction model where some demand after interdiction can be outsourced at some cost [1], an interdiction problem with hierarchical system of facilities [7], a more general median protection/interdiction problem that involves outsourcing, capacitated nested hierarchical facilities, and the budget of interdiction [14], etc.

The aforementioned fortification-interdiction models assume that there is an existing service system and the goal is to thwart intentional attacks by protecting some facilities. Another approach consists in taking into account possible worst-case facility losses in the initial location of facilities. Such problems, known as location-interdiction models [29], are not widely addressed in the literature. For example, in [23], the authors extend the $r$-interdiction covering problem from [11] and introduce a maximal covering location-interdiction problem aimed at maximizing the coverage of customers if the most critical facilities are failed. Note that location-interdiction problems are more difficult to solve than the protection-interdiction ones and most of effective algorithms developed for protection problems are not applicable to them [29]. Other closely related problems are aimed at combining both location and protection decisions. An example is the model proposed in [2], where capacitated facilities may be located either protected or unprotected. In [3] the problem is to first locate a given number of facility followed by protecting some of them with a limited protection budget.

In this paper we propose a new facility location-interdiction model by extending the $r$-interdiction median problem in the way closely related to [23]. We formulate this problem as a bilevel integer linear program where the upper-level player (defender) finds $p$ sites for locating facilities in order to minimize the overall cost of serving customers, taking into account that the lower-level player (attacker) hits some $r$ facilities in order to inflict the maximal possible damage. We demonstrate that this problem can be reformulated as a single-level integer program so that small- and medium size problem instances can be solved with a general purpose commercial solver. For a particular case when the attacker hits a single open facility, we develop a fast local search heuristic.

\section{Bilevel Facility Location-Interdiction Problem}

Our model relies upon the uncapacitated $p$-median problem. It is one of the basic and widely studied facility location problems. It has been applied in designing service and distribution systems in private and public sectors [20]. It is also a powerful modeling tool with applications to production $[9,21]$ and machine learning $[16,30]$. 
We suppose that there is a set $I(|I|=m)$ of potential facility location sites and a set $J(|J|=n)$ of customers. We denote as $d_{i j}$ the shortest distance (service cost) between a customer $j$ and the facility located at cite $i$. As for the p-median problem, each customer is served by the closest open facility. If for a customer, the closest open facility is interdicted, she/he is reassigned to the closest open non-interdicted facility. The interdicted facilities become completely unavailable. As the attacker has some limited resources for interdiction, we assume that only $r$ out of $p$ open facilities can be attacked. Note that we suppose that the attacker has perfect information about where the facilities are located, hence he/she always hits only open facilities. We also suppose that all $r$ facilities chosen by the interdictor are attacked simultaneously, thus the defender has no way to thwart any losses.

Our problem can be viewed as a static Stackelberg game where two players make sequential decisions. The first player (leader or defender) first decides where to locate $p$ facilities in order to minimize the overall sum of distances between customers and their closest open facilities, taking into account that some facilities may be interdicted; the second player (follower or attacker), knowing where the first player has located the facilities, tries to inflict the maximal possible harm to the service system by attacking $r$ of them. Thus, some customers have to be reassigned to more distant facilities, which reduces the system efficiency.

Let us introduce the following decision variables:

$$
y_{i}= \begin{cases}1, & \text { if a facility is located at site } \mathrm{i} \\ 0, & \text { otherwise }\end{cases}
$$

$$
\begin{gathered}
x_{i j}= \begin{cases}1, & \text { if a customer } \mathrm{j} \text { is assigned to the facility located at site } \mathrm{i} ; \\
0, & \text { otherwise }\end{cases} \\
s_{i}= \begin{cases}1, & \text { if a facility located at site } \mathrm{i} \text { is interdicted; } \\
0, & \text { otherwise, }\end{cases}
\end{gathered}
$$

Furthermore, to ensure the assignment of customers to the closest facilities, we define the set $W_{i j}=\left\{k \in I: d_{k j}>d_{i j}\right\}$, i.e. the set of potential sites which are farther from customer $j$ than site $i$. The bilevel interdiction-location median problem can be written as follows:

$$
\begin{gathered}
\min \sum_{i \in I} \sum_{j \in J} d_{i j} x_{i j}^{*}(y), \\
\sum_{i \in I} y_{i}=p, \\
y_{i} \in\{0,1\}
\end{gathered}
$$


where $x_{i j}^{*}(y)$ is a solution of the attacker's problem:

$$
\begin{array}{lr}
\max \sum_{i \in I} \sum_{j \in J} d_{i j} x_{i j}, & \\
\sum_{i \in I} x_{i j}=1 & \forall j \in J, \\
x_{i j} \leq y_{i} & \forall i \in I, j \in J, \\
\sum_{i \in I} s_{i}=r, & \\
s_{i} \leq y_{i} & \forall i \in I, \\
\sum_{k \in W_{i j}} x_{k j} \leq 1+s_{i}-y_{i} & \forall i \in I, j \in J, \\
s_{i}, x_{i j} \in\{0,1\} & \forall i \in I, j \in J .
\end{array}
$$

Note that the defender and the attacker has the opposite objectives. The system planner attempts to locate $p$ facilities to minimize the overall sum of distances between customers and the facilities remained after eliminating $r$ of them. The interdictor determines which open facilities to hit in order to maximize the cost of serving customers. Constraints (2) set the number of facilities opened by the defender. Constraints (5) and (6) are the standard facility location constraints guaranteeing that each customer is served by exactly one open facility. Constraints (7) and (8) impose that the attacker can hit only $r$ out of $p$ open facilities. Finally, constraints (9) ensure that each customer is served from the closest open facility after attack occurs. Indeed, if a facility is located at site $i$ and not interdicted, a customer $j$ cannot be served by a more distant facility than $i$. As was noted in [27], these constraints can also be adapted to settings where customers are not supposed to be served from the closest open facilities. Instead, they may have some facilities preference ordering known for both the defender and the attacker (e.g. see [31-33]).

Bilevel programming problems are usually very hard to solve, especially when the problems on both levels are mixed-integer programs. In facility location, bilevel problems usually arise in competitive location when there are two players that compete for serving the demand of customers from a market [17]. A good example of competitive facility location problems is the well-known $(r \mid p)$-centroid problem $[6,12]$. This problem is known to be $\Sigma_{2}^{P}$ hard [13].

As was noted above, the location-interdiction problems are much more challenging than widely studied fortification-interdiction problems. Their structure hinders from application some very efficient exact approaches based on implicit enumeration and decomposition techniques.

\section{Integer Programming Formulation}

In this section we demonstrate that the introduced location-interdiction median problem can be formulated as a single-level integer program. Thus, it can be solved by a commercial branch-and-bound solver. 
For our formulation, we denote by $H$ the set of all interdiction patterns, i.e. it consists of all possible strategies of interdicting $r$ out of $m$ potential location sites. We also denote by $h$ the index corresponding to a specific interdiction strategy and by $I_{h}$ the set of interdicted sites in pattern $h$. Our formulation uses the variables $y_{i}$ that have the same definition as those in formulation (1)-(10). We also introduce the following binary variables:

$$
x_{i j}^{h}= \begin{cases}1, & \text { if a customer } \mathrm{j} \text { is assigned to the facility located at site } \mathrm{i} \\ & \text { when interdiction pattern h occurs; } \\ 0, & \text { otherwise }\end{cases}
$$

Thus, we can formulate our problem as follows:

$$
\begin{array}{lr}
\min z & \\
z \geq \sum_{i \in I} \sum_{j \in J} d_{i j} x_{i j}^{h} & h \in H, \\
\sum_{i \in I} x_{i j}^{h}=1 & \forall j \in J, h \in H, \\
& x_{i j}^{h} \leq y_{i} \\
\sum_{i \in I} y_{i}=p, & \forall i \in I, j \in J, h \in H, \\
& \sum_{i \in I_{h}} \sum_{j \in J} x_{i j}^{h}=0, \\
y_{i} \in\{0,1\} & \forall h \in H, \\
x_{i j}^{h} \in\{0,1\} & \forall i \in I, j \in J, h \in H .
\end{array}
$$

The objective function (11) is to minimize the overall cost $z$ of serving customers from operational facilities remained after the worst-case attack on $r$ potential location sites. Constraints (13)-(14) have the same definition as those in formulation (1)-(10), i.e. they ensure that each customer $j$ must be assigned only to one open facility when interdiction pattern $h$ occurs. Constraints (12) define the cost of serving customers from a given set of facilities if $r$ sites corresponding to pattern $h$ are lost. Note that given a set of facilities, if none of them are located at the sites from interdiction pattern $h$, then the cost $z$ is equal to the $p$-median objective value. Finally, constraints (16) ensure that customers cannot be served by facilities located at the sites interdicted in pattern $h$.

Note that the model actually involves the exponential number of variables and constraints. However, the common assumption is that terrorist groups usually have limited resources and are able to simultaneously hit only a small number of facilities [27]. In this case, one can determine all possible $\left(\begin{array}{c}m \\ r\end{array}\right)$ interdiction patterns in advance. In particular, if only one facility is supposed to be attacked, the model contains $\mathcal{O}\left(m^{2} n\right)$ decision variables and constraints. 


\section{Local Search}

Bilevel integer problems are quite challenging for exact methods, especially in case of real-life instances of large size. Thus, the development of heuristics to find quality feasible solutions in reasonable time is quite natural. The main idea is to use a local search procedure which attempts to improve an initial solution by searching its neighbors. The location-interdiction median problem is based on the well-known $p$-median problem, hence the simplest and natural strategy is to develop a local search procedure over the SWAP neighborhood. It consists of all defender's solutions which are obtained from a current incumbent by closing one facility and opening another one in a different place.

In order to compute the defender's objective value for a given set of open facilities (the set of variables $y_{i}$ ), we actually have to find an optimal solution of the attacker's problem:

$$
\begin{aligned}
& \max \sum_{i: y_{i}=1} \sum_{j \in J} d_{i j} x_{i j}, \\
& \sum_{i: y_{i}=1} x_{i j}=1 \text {, } \\
& j \in J, \\
& \sum_{k \in T_{i j}(y)} x_{k j} \leq s_{i}, \quad i \in I: y_{i}=1, j \in J, \\
& \sum_{i: y_{i}=1} s_{i}=r \text {, } \\
& s_{i}, x_{i j} \in\{0,1\} \text {, }
\end{aligned}
$$

where $T_{i j}(y)$ is the set of open defender's facilities which are farther from customer $j$ than facility $i$. This problem can be solved by a commercial branch-andbound solver. On the other hand, in some applications where the numbers of both open and interdicted facilities are small, the attacker's problem can effectively be solved by enumerating all $\left(\begin{array}{l}p \\ r\end{array}\right)$ possible facility losses.

In this section we focus on a special case of the attacker's problem where $r=1$. Our main goal is to develop a local search procedure which is faster than the naive implementation based on explicit enumeration.

Throughout this section we use the following notations. We denote as $d(i, j)$ the distance (service cost) between a customer $j$ and a location $i$. We suppose that $S$ is a solution of the defender's problem, i.e. $S$ is any subset of $I$ consisting of $p$ elements. The closest open facility in $S$ for customer $j$ is denoted as $c_{1}(j)$. To perform a fast interchange, our algorithm needs the second and the third open facilities to $j$ that we denote as $c_{2}(j)$ and $c_{3}(j)$, respectively. Searching the swap neighborhood, the algorithm picks one candidate facility to add to the current solution and one to drop from it that we refer to as $f_{i}$ and $f_{r}$, respectively. Following [25], we assume that the distance between any facility site and any customer can be computed in $\mathcal{O}(1)$ time. For example, this is the case when the distance matrix is already calculated. 
Note that for the $p$-median problem, there are several fast implementations of the swap-based local search, including prominent Whitaker's implementation [34] and a fast implementation from [25].

Given a solution $S$, we need to find a neighbor $\hat{S}$ providing a better value of the defender's objective or to check that such an improving neighbor does not exist. Thus, we have $p$ sites for dropping and $m-p$ sites for insertion. Given a pair $\left(f_{i}, f_{r}\right)$, we have to compute the gain of swapping $f_{r}$ with $f_{i}$. A straightforward implementation assumes that, for each possible swap $\left(f_{i}, f_{r}\right)$, we have to solve the attacker's problem, i.e. to enumerate all possible strategies of interdicting facilities in the solution obtained after swapping. If $r=1$, the number of interdiction patterns is equal to $p$. If the three closest open facilities are not pre-computed and the calculation of distances takes $\mathcal{O}(1)$ time, determining the assignment of all customers under each interdiction pattern takes $\mathcal{O}(p n)$ time. Since the total number of swaps is equal to $p(m-p)=\mathcal{O}(p m)$ and the number of interdiction patterns for each possible swap is $p$, one iteration of local search takes $\mathcal{O}\left(p^{3} m n\right)$ time. Suppose now that the three closest facilities to each customer $j$ are found: this takes $\mathcal{O}(p n)$ time. In this case, one iteration of local search can be performed in $\mathcal{O}\left(p^{2} m n\right)$ time.

However, the computation time can be further reduced by avoiding the explicit enumeration needed to find an optimal solution of the attacker's problem. Indeed, the value of the defender's objective function $z^{\text {lead }}$ for a solution $S$ can be written as follows:

$$
z^{\text {lead }}=z^{\text {med }}+\max _{s \in S} \sum_{j: c_{1}(j)=s} d\left(c_{2}(j), j\right)-d\left(c_{1}(j), j\right),
$$

where $z^{\text {med }}$ is the value of the p-median objective. In other words, the cost that the defender will get after a disruptive strike can be computed as the sum of costs for serving customers from the closest open facilities plus the cost induced by the customers whose closest facility was lost due to attack. Thus, for each pair $\left(f_{i}, f_{r}\right)$, we must compute the gain from swapping $f_{i}$ and $f_{r}$ according to both the $p$-median objective and the value induced by attack:

$$
\operatorname{cost}\left(f_{i}, f_{r}\right)=\operatorname{med} \operatorname{cost}\left(f_{i}, f_{r}\right)+\operatorname{losscost}\left(f_{i}, f_{r}\right),
$$

where medcost $\left(f_{i}, f_{r}\right)$ is computed as follows:

$$
\begin{aligned}
\operatorname{medcost}\left(f_{i}, f_{r}\right)= & \sum_{j: c_{1}(j) \neq f_{r}} \min \left\{0, d\left(f_{i}, j\right)-d\left(c_{1}(j), j\right)\right\} \\
& +\sum_{j: c_{1}(j)=f_{r}}\left(\min \left\{d\left(c_{2}(j), j\right), d\left(f_{i}, j\right)\right\}-d\left(c_{1}(j), j\right)\right) .
\end{aligned}
$$

In other words, if the closest facility of a customer $j$ is not replaced (it is not $f_{r}$ ), the customer either reassigns to $f_{i}$, if it is closer, or stays assigned to the current facility. If customer $j$ is served from $f_{r}$, then he/she assigns to the closest open facility: $f_{i}$ or $c_{2}(j)$. If medcost $\left(f_{i}, f_{r}\right)<0$, then we can gain from such a swap with respect to the $p$-median objective. 
The value of $\operatorname{losscost}\left(f_{i}, f_{r}\right)$ can be computed in a similar way (see Algorithm 1).

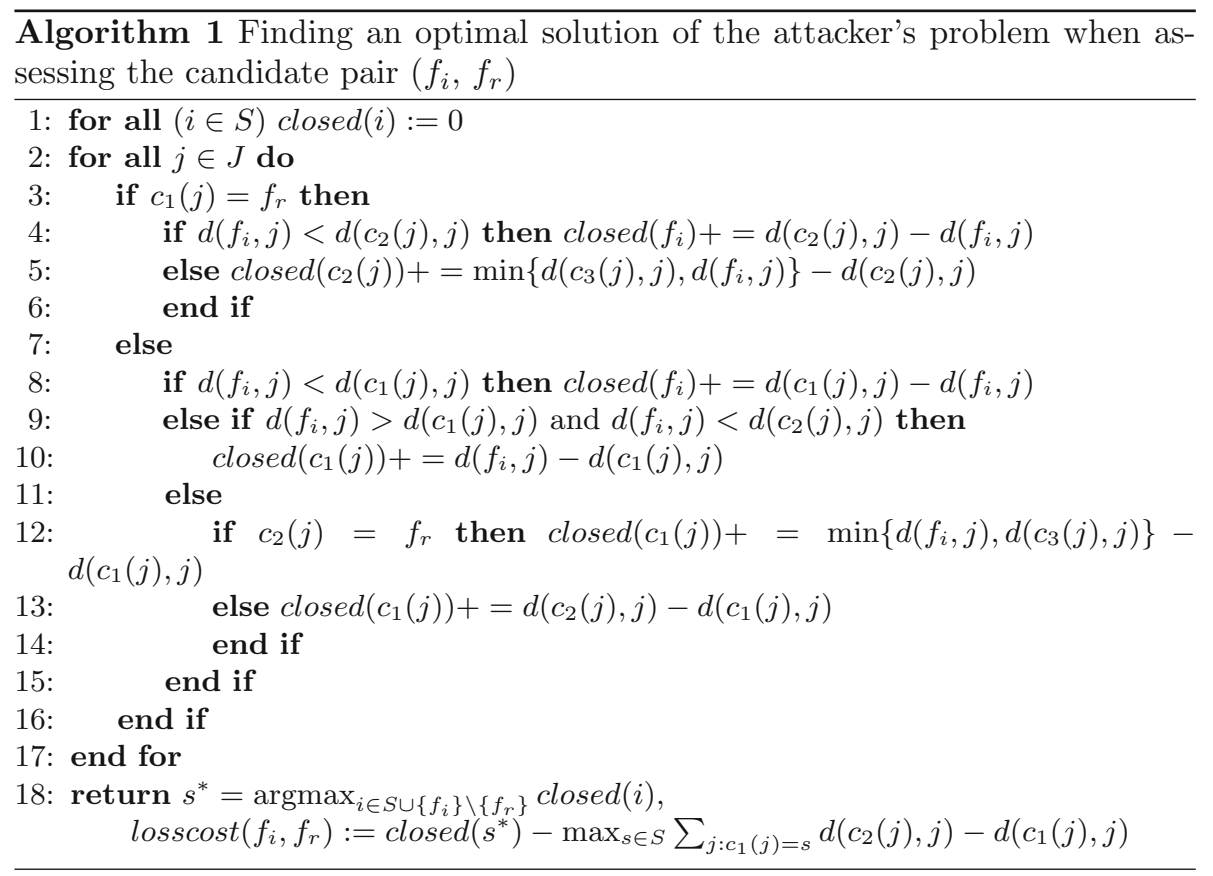

The main idea is to implicitly calculate the costs of all interdiction patterns, considering each customer $j$ independently. Note that the attacker can cause a harm to customer $j$ if he hits the closest open facility to $j$.

If the closest facility to $j$ is not dropped, $f_{i}$ may be either the closest to $j$ or the second closest. In the first case, the attacker may inflict damage only by hitting $f_{i}$, hence $j$ remains assigned to the same facility (she/he was assigned before swapping). In the second case, the attacker striking the closest facility $c_{1}(j)$ forces the customer be served from the next facility: $f_{i}$. There is also another option. The candidate $f_{i}$ may also be farther than the second closest facility $c_{2}(j)$. In this case, if $c_{2}(j)$ is dropped, the attacker engenders a harm by hitting the closest facility $c_{1}(j)$ and forcing the customer assign to the third closest facility $c_{3}(j)$. Otherwise, if $c_{2}(j)$ is not removed, the customer assigns to it in case of attack.

If the closest facility $c_{1}(j)$ is dropped, there are two options. The new facility $f_{i}$ is closer than $c_{2}(j)$, thus an attack on $f_{i}$ results in assigning the customer to the second closest facility $c_{2}(j)$. If the new facility is farther than $c_{2}(j)$, the customer in case of attack on $c_{2}(j)$ would be served from the third closest facility: $c_{3}(j)$ or $f_{i}$.

In all cases described above, for each customer $j$, only an attack on the closest facility (after swapping) can leads to reassigning the customer to the 
next closest facility. Thus, closed in Algorithm 1 accumulates the contribution of each particular customer to the cost of all possible interdiction patterns.

The values of medcost $\left(f_{i}, f_{r}\right)$ and $\operatorname{losscost}\left(f_{i}, f_{r}\right)$ can obviously be computed in $\mathcal{O}(n)$ time for each candidate pair. As the total number of swaps is $p(m-$ $p)$, each iteration of local search takes $\mathcal{O}(p m n)$ time. Note that the standard implementation of swap-based local search for the $p$-median problem requires the same time per iteration. After a swap is accepted, updating the closest facilities for each customer requires $\mathcal{O}(p n)$ time in the worst case.

\section{Computational Experiments}

In this section we report some computational experiments to test the proposed integer linear program as well as the local search procedure devised. We utilized several widely used test problem instances from the well-known TSPLIB ${ }^{1}$. Our integer linear model was set up using IBM ILOG Concert technology and C++ programming language. It was then solved with IBM ILOG CPLEX 12.8.0², freely available for non-commercial research. We implemented the local search procedure using $\mathrm{C}++$ programming language as well. Note that our local search is not parallel, while CPLEX was allowed to use four parallel threads. We suppose that the number of interdictions $r$ is equal to 1 . The initial solutions for the local search were chosen at random. We performed only one run of the local search on each particular test problem.

The results are presented in Table 1, where the first column shows the problem name, column $p$ contains the number of defender's facilities to be located. In Objective values, we report three following values. Column Pmed shows the optimal value of the p-median objective, i.e. the service cost without the threat of possible attacks. Columns $\operatorname{Intr} C P L X$ and $L S$ demonstrate the values of the defender's objective function found by CPLEX and the local search, respectively. Columns Time cplx $_{\text {and }}$ Time $_{L S}$ represent the running times of CPLEX and the local search.

We can observe that CPLEX requires very much time to find an optimal solution of the single-level location-interdiction median problem. For example, CPLEX spent more than almost two hours to solve berlin52 instance involving 52 customers and 5 facilities. Recall that the single-level problem contains very large number of variables and constraints, which makes it much harder than the underlying $p$-median problem.

Observe that the found objective values of the location-interdiction problem are in general much larger than those of the $p$-median problem, especially when the number of facilities is small. For example, for the problem berlin52, incorporating risks of a possible deliberate attack when locating 3 facilities results in increasing the service cost for over $27 \%$ with respect to the cost of the location strategy that does not assume facility losses. Thus, if the system planner is riskaverse, she may attempt to mitigate the impact of an attack but it may be quite

\footnotetext{
${ }^{1}$ http://elib.zib.de/pub/mp-testdata/tsp/tsplib.

${ }^{2}$ https://www.ibm.com/products/ilog-cplex-optimization-studio.
} 
Table 1. Results on the TSPLIB problem instances, where $p$ is the number of facilities opened by the defender, the number of interdictions $r=1$.

\begin{tabular}{|c|c|c|c|c|c|c|c|}
\hline \multirow[b]{2}{*}{ Problem } & \multicolumn{5}{|c|}{ Objective values } & \multirow[t]{2}{*}{ Time $_{c p l x}$} & \multirow[t]{2}{*}{ Time $_{L S}$} \\
\hline & $\mathrm{m}$ & $\mathrm{p}$ & Pmed & IntrCPLX & LS & & \\
\hline \multirow[t]{4}{*}{ ulysses 22} & 22 & 2 & 70.02 & 123.47 & 123.47 & 7.1 & 0 \\
\hline & & 3 & 51.55 & 109.74 & 109.74 & 12.5 & 0 \\
\hline & & 4 & 42.57 & 67.54 & 67.54 & 9.5 & 0 \\
\hline & & 5 & 35.30 & 54.13 & 54.13 & 4.92 & 0 \\
\hline \multirow[t]{4}{*}{ bayg29 } & 29 & 2 & 13601.85 & 19469.92 & 19469.92 & 43.5 & 0 \\
\hline & & 3 & 10446.36 & 15343.86 & 16731.25 & 57.0 & 0 \\
\hline & & 4 & 9013.30 & 12180.74 & 12708.10 & 57.5 & 0 \\
\hline & & 5 & 7735.65 & 10239.33 & 10239.33 & 55.7 & 0.001 \\
\hline \multirow[t]{4}{*}{ att48 } & 48 & 2 & 72921.90 & 113222.34 & 113222.34 & 1262.3 & 0.001 \\
\hline & & 3 & 54623.16 & 85741.06 & 86337.62 & 2641.2 & 0.001 \\
\hline & & 5 & 39679.31 & 53308.19 & 53308.19 & 3854.7 & 0.001 \\
\hline & & 8 & 29159.05 & 35615.89 & 35615.89 & 2864.9 & 0.002 \\
\hline \multirow[t]{4}{*}{ berlin52 } & 52 & 2 & 14816.78 & 20000.29 & 20000.29 & 2825.8 & 0.001 \\
\hline & & 3 & 12057.82 & 16604.35 & 17599.84 & 4081.5 & 0.001 \\
\hline & & 5 & 8888.74 & 12158.69 & 12199.40 & 7125.8 & 0.002 \\
\hline & & 8 & 6402.17 & 8081.50 & 8080.88 & 5182.7 & 0.002 \\
\hline \multirow[t]{3}{*}{ fl1400 } & 1400 & 50 & 29090.23 & - & 31627.20 & - & 80.6 \\
\hline & & 100 & 16552.22 & - & 17500.34 & - & 264.3 \\
\hline & & 200 & 9355.34 & - & 10230.72 & - & 654.6 \\
\hline
\end{tabular}

costly. Note that in case when 8 facilities are located, the increase in service cost is about $20 \%$. However, for the problem fl1400, the increase is only $9 \%$ if 200 facilities are located. Thus, when the number of open facilities is small, the system planner may prefer a risky strategy (locating facilities according to the $p$-median protocol) rather than the robust design of a service system resulting in increase in the service cost. On the other hand, when the number of facilities is relatively large, a robust system can be designed with small increase in the service cost.

We see that the local search procedure found the same solutions as CPLEX in most cases (especially for small problem instances). Recall that we ran the local search only once. For berlin $52, p=8$, it found a solution that even a little better. Note that this may happen due to a relatively large duality gap inherent in the single-level integer program.

Observe that the proposed heuristic is very fast, especially in comparison with the commercial solver. It spent less than one second to find very close to optimal solutions. 


\section{Conclusion}

In this paper we proposed a new location-interdiction median problem based on the well-known p-median problem. This problem can naturally be formulated as a bilevel integer linear program where the system designer makes decision about where to locate facilities; and the attacker interdicts some facilities to engender the maximal harm to the system efficiency. We demonstrated that the problem can be formulated as a single-level integer linear problem that can be solved with a commercial solver. Since it can be utilized to solving only small problem instances, even assuming that only one facility is interdicted, we developed a fast implementation of local search over the basic SWAP-neighborhood.

Our further research may be focused on developing alternative formulations of the problem as well as exact solution approaches (branch-and-cut methods). Our research will also aim at extending the proposed technique for larger values of $r$ and developing new heuristics. Finally, the developed local search procedures may be employed to devise various metaheuristics.

Acknowledgement. The reported study was funded by RFBR according to the research project No. 18-07-01037.

\section{References}

1. Aksen, D., Akca, S.S., Aras, N.: A bilevel partial interdiction problem with capacitated facilities and demand outsourcing. Comput. Oper. Res. 41, 346-358 (2014). https://doi.org/10.1016/j.cor.2012.08.013

2. Aksen, D., Aras, N.: A bilevel fixed charge location model for facilities under imminent attack. Comput. Oper. Res. 39(7), 1364-1381 (2012). https://doi.org/ 10.1016/j.cor.2011.08.006

3. Aksen, D., Aras, N., Piyade, N.: A bilevel p-median model for the planning and protection of critical facilities. J. Heuristics 19(2), 373-398 (2013). https://doi. org/10.1007/s10732-011-9163-5

4. Aksen, D., Piyade, N., Aras, N.: The budget constrained r-interdiction median problem with capacity expansion. Cent. Eur. J. Oper. Res. 18, 269-291 (2010). https://doi.org/10.1007/s10100-009-0110-6

5. Al Jazeera: Weakest link: Global supply chains disrupted by coronavirus (2020). https://www.aljazeera.com/ajimpact/weakest-link-global-supply-chainsdisrupted-due-coronavirus-200212033618174.html

6. Alekseeva, E., Kochetov, Y., Plyasunov, A.: An exact method for the discrete $(r \mid p)$-centroid problem. J. Glob. Optim. 63(3), 445-460 (2013). https://doi.org/ 10.1007/s10898-013-0130-6

7. Aliakbarian, N., Dehghanian, F., Salari, M.: A bi-level programming model for protection of hierarchical facilities under imminent attacks. Comput. Oper. Res. 64, 210-224 (2015). https://doi.org/10.1016/j.cor.2015.05.016

8. Automative News Europe: Nissan faces global parts shortage due to coronavirus, report says (2020). https://europe.autonews.com/automakers/nissan-faces-globalparts-shortage-due-coronavirus-report-says 
9. Avella, P., Boccia, M., Martino, C.D., Oliviero, G., Sforza, A., Vasilyev, I.: A decomposition approach for a very large scale optimal diversity management problem. 4OR 3(1), 23-37 (2005). https://doi.org/10.1007/s10288-004-0059-1

10. Church, R.L., Scaparra, M.P.: Protecting critical assets: the r-interdiction median problem with fortification. Geogr. Anal. 39(2), 129-146 (2007). https://doi.org/ 10.1111/j.1538-4632.2007.00698.x

11. Church, R.L., Scaparra, M.P., Middleton, R.S.: Identifying critical infrastructure: the median and covering facility interdiction problems. Ann. Am. Assoc. Geogr. 94(3), 491-502 (2004). https://doi.org/10.1111/j.1467-8306.2004.00410.x

12. Davydov, I., Kochetov, Y., Carrizosa, E.: A local search heuristic for the $(r \mid p)$ centroid problem in the plane. Comput. Oper. Res. 52, 334-340 (2014). https://doi. org/10.1016/j.cor.2013.05.003. Recent advances in Variable neighborhood search

13. Davydov, I., Kochetov, Y., Plyasunov, A.: On the complexity of the $(r-p)$-centroid problem in the plane. TOP 22(2), 614-623 (2013). https://doi.org/10.1007/s11750013-0275-y

14. Forghani, A., Dehghanian, F., Salari, M., Ghiami, Y.: A bi-level model and solution methods for partial interdiction problem on capacitated hierarchical facilities. Comput. Oper. Res. 114, 104831 (2020). https://doi.org/10.1016/j.cor.2019. 104831

15. Ghaffarinasab, N., Atayi, R.: An implicit enumeration algorithm for the hub interdiction median problem with fortification. Eur. J. Oper. Res. 267(1), 23-39 (2018). https://doi.org/10.1016/j.ejor.2017.11.035

16. Hansen, P., Jaumard, B.: Cluster analysis and mathematical programming. Math. Program. 79(1-3), 191-215 (1997)

17. Iellamo, S., Alekseeva, E., Chen, L., Coupechoux, M., Kochetov, Y.: Competitive location in cognitive radio networks. 4OR 13(1), 81-110 (2014). https://doi.org/ 10.1007/s10288-014-0268-1

18. Israeli, E., Wood, R.K.: Shortest-path network interdiction. Networks 40(2), 97111 (2002). https://doi.org/10.1002/net.10039

19. Liberatore, F., Scaparra, M.P., Daskin, M.S.: Analysis of facility protection strategies against an uncertain number of attacks: the stochastic r-interdiction median problem with fortification. Comput. Oper. Res. 38(1), 357-366 (2011). https:// doi.org/10.1016/j.cor.2010.06.002

20. Marianov, V., Serra, D.: Median problems in networks. In: Eiselt, H.A., Marianov, V. (eds.) Foundations of Location Analysis. ISORMS, vol. 155, pp. 39-59. Springer, New York (2011). https://doi.org/10.1007/978-1-4419-7572-0_3

21. Masone, A., Sterle, C., Vasilyev, I., Ushakov, A.: A three-stage p-median based exact method for the optimal diversity management problem. Networks $\mathbf{7 4}(2)$, 174-189 (2019). https://doi.org/10.1002/net.21821

22. McMasters, A.W., Mustin, T.M.: Optimal interdiction of a supply network. Nav. Res. Logist. 17(3), 261-268 (1970). https://doi.org/10.1002/nav.3800170302

23. O'Hanley, J.R., Church, R.L.: Designing robust coverage networks to hedge against worst-case facility losses. Eur. J. Oper. Res. 209(1), 23-36 (2011). https://doi.org/ 10.1016/j.ejor.2010.08.030

24. Quadros, H., Roboredo, M.C., Pessoa, A.A.: A branch-and-cut algorithm for the multiple allocation r-hub interdiction median problem with fortification. Expert Syst. Appl. 110, 311-322 (2018). https://doi.org/10.1016/j.eswa.2018.05.036

25. Resende, M., Werneck, R.: A fast swap-based local search procedure for location problems. Ann. Oper. Res. 150, 205-230 (2007). https://doi.org/10.1007/s10479006-0154-0 
26. Roboredo, M.C., Pessoa, A.A., Aizemberg, L.: An exact approach for the rinterdiction median problem with fortification. RAIRO-Oper. Res. 53(2), 505-516 (2019). https://doi.org/10.1051/ro/2017060

27. Scaparra, M.P., Church, R.L.: A bilevel mixed-integer program for critical infrastructure protection planning. Comput. Oper. Res. 35(6), 1905-1923 (2008). https://doi.org/10.1016/j.cor.2006.09.019

28. Scaparra, M.P., Church, R.L.: An exact solution approach for the interdiction median problem with fortification. Eur. J. Oper. Res. 189(1), 76-92 (2008). https://doi.org/10.1016/j.ejor.2007.05.027

29. Scaparra, M.P., Church, R.L.: Location problems under disaster events. In: Laporte, G., Nickel, S., Saldanha da Gama, F. (eds.) Location Science, pp. 631-656. Springer, Cham (2019). https://doi.org/10.1007/978-3-030-32177-2_22

30. Sidorov, D., Wei, W.S., Vasil'ev, I., Salerno, S.: Automatic defects classification with p-median clustering technique. In: 2008 10th International Conference on Control, Automation, Robotics and Vision, pp. 775-780. IEEE, Piscataway (2008). https://doi.org/10.1109/ICARCV.2008.4795615

31. Vasil'ev, I.L., Klimentova, K.B., Kochetov, Y.A.: New lower bounds for the facility location problem with clients' preferences. Comput. Math. Math. Phys. 49(6), 1010-1020 (2009). https://doi.org/10.1134/S0965542509060098

32. Vasilyev, I., Klimentova, X., Boccia, M.: Polyhedral study of simple plant location problem with order. Oper. Res. Lett. 41(2), 153-158 (2013). https://doi.org/10. 1016/j.orl.2012.12.006

33. Vasilyev, I.L., Klimentova, K.B.: The branch and cut method for the facility location problem with client's preferences. J. Appl. Ind. Math. 4(3), 441-454 (2010). https://doi.org/10.1134/S1990478910030178

34. Whitaker, R.A.: A fast algorithm for the greedy interchange for large-scale clustering and median location problems. INFOR 21, 95-108 (1983). https://doi.org/ 10.1080/03155986.1983.11731889

35. Zhang, P., Fan, N.: Analysis of budget for interdiction on multicommodity network flows. J. Glob. Optim. 67(3), 495-525 (2016). https://doi.org/10.1007/s10898-0160422-8

36. Zheng, K., Albert, L.A.: An exact algorithm for solving the bilevel facility interdiction and fortification problem. Oper. Res. Lett. 46(6), 573-578 (2018). https:// doi.org/10.1016/j.orl.2018.10.001 